\title{
Reprint of "Affective picture processing as a function of preceding picture valence: An ERP analysis"
}

\author{
Harald T. Schupp ${ }^{a, *}$, Ralf Schmälzle ${ }^{a}$, Tobias Flaisch ${ }^{a}$, Almut I. Weike ${ }^{b}$, Alfons O. Hamm ${ }^{b}$ \\ ${ }^{a}$ Department of Psychology, University of Konstanz, Germany \\ ${ }^{\mathrm{b}}$ Department of Psychology, University of Greifswald, Germany
}

\begin{abstract}
A B S T R A C T
Event-related brain potential (ERP) studies consistently revealed that a relatively early (early posterior negativity; EPN) and a late (late positive potential; LPP) ERP component differentiate between emotional and neutral picture stimuli. Two studies examined the processing of emotional stimuli when preceded either by pleasant, neutral, or unpleasant context images. In both studies, distinct streams of six pictures were shown. In Study 1, hedonic context was alternated randomly across the 180 picture streams. In Study 2 , hedonic context sequences were blocked, resulting in 60 preceding sequences of pleasant, neutral, and unpleasant context valence, respectively. The main finding was that the valence of the preceding picture sequence had no significant effect on the emotional modulation of the EPN and LPP components. However, previous results were replicated in that emotional stimulus processing was associated with larger EPN and LPP components as compared to neutral pictures. These findings suggest that the prioritized processing of emotional stimuli is primarily driven by the valence of the current picture.
\end{abstract}

\section{Introduction}

In a world where various stimuli compete for attentional resources, the fast and reliable detection of positive and negative reinforcers facilitates adaptive behavior (Lang et al., 1997; Öhman et al., 2000). Thus, functional and evolutionary considerations suggest the preferential processing of emotional cues. Over the past decade, numerous neuroimaging studies have confirmed that emotional cues guide selective visual attention. Functional magnetic resonance imaging (fMRI) revealed increased BOLD (blood oxygen level dependent) signals in associative visual regions (extrastriate, occipito-parietal, and inferior temporal cortex) and subcortical limbic structures when viewing emotionally arousing pictures compared to neutral pictures (e.g., Bradley et al., 2003; Costafreda et al., 2008; Junghöfer et al., 2006; Sabatinelli et al., 2007, 2011). Furthermore, event-related brain potential (ERP) studies revealed the temporal dynamics of emotion processing at the level of distinct processing stages and demonstrated that emotional stimuli receive enhanced processing early in the processing stream (Schupp et al., 2006).

Two ERP components, referred to as early posterior negativity (EPN) and late positive potential (LPP), have consistently been

DOI of original article: http://dx.doi.org/10.1016/j.biopsycho.2012.04.006.

* Corresponding author at: Institute of Psychology, University of Konstanz, PO Box D 36, 78457 Konstanz, Germany. Tel.: +49 7531 882504; fax: +49 7531882971.

E-mail address: Harald.Schupp@uni-konstanz.de (H.T. Schupp). found to be modulated by emotional picture valence. The EPN component is observed as a relative negative difference in processing emotional pictures (pleasant and unpleasant) compared to neutral pictures over temporo-occipital sites in a time window between 150 and 350 ms (e.g., Junghöfer et al., 2001; Schupp et al., 2003, 2006). The late positive potential, measured over centro-parietal regions between 300 and $700 \mathrm{~ms}$, is larger for emotional, compared to neutral, stimuli (e.g., Palomba et al., 1997; Schupp et al., 2000; Sabatinelli et al., 2007). Studies relying on stimuli from the International Affective Picture System (IAPS, Lang et al., 2008) observed that EPN and LPP modulations are most accentuated for pictures depicting evolutionarily relevant stimulus contents, such as reproduction- and defense-related scenes (Schupp et al., 2003, 2006), which also provoke reliable somatic, autonomic and humoral responses (Bradley et al., 2001). Recent research demonstrated the emotional modulation of these ERP components across a broader array of emotional stimuli including emotional facial expressions (Mühlberger et al., 2009; Sato et al., 2001; Schupp et al., 2004; Wieser et al., 2010), emotional words (Kissler et al., 2006), symbolic gestures (Flaisch et al., 2009, 2011), and clashing moral statements (Van Berkum et al., 2009). These findings have been considered from the perspective of 'natural selective attention', which holds that under naturalistic conditions stimulus perception and evaluation are often directed by underlying motivational systems of avoidance and approach (Bradley, 2009; Lang et al., 1997; Schupp et al., 2006).

Previous studies have mostly presented emotional stimuli in temporal isolation and used carefully planned presentation 
schemes to avoid confounds due to sequence effects (Flaisch et al., 2008a,b). However, in the natural environment, stimuli typically do not occur in isolation; instead, emotional stimuli may cluster in time and space. Accordingly, people may encounter congruent streams or contexts of pleasant or unpleasant stimuli. This raises the question of how the repeated engagement of either the appetitive or aversive motivational system affects the processing of individual emotional cues. One recent study addressed this issue by presenting emotional IAPS pictures either in an intermixed fashion or as streams of 8 pictures of the same stimulus type (Pastor et al., 2008). Results indicate that emotional modulation of the LPP and positive slow wave was comparable in both mixed and repeated presentation conditions. On the other hand, research using a modified oddball paradigm suggests that hedonic contexts may indeed modulate affective stimulus processing. For instance, positive and negative personality adjectives elicit larger LPP amplitudes when presented in a sequence of adjectives of opposite valence contexts than same valence contexts (Cacioppo et al., 1993, 1994; Crites et al., 1995). A number of methodological differences may account for the divergent findings obtained in previous studies. For instance, studies differed with regard to the type of emotional stimuli (words vs. pictures) or the duration of stimulus presentation ( 1.5 vs. $12 \mathrm{~s}$ ). Further research is thus needed to elucidate the effects of hedonic contexts upon emotional stimulus processing. Accordingly, the present study investigated the processing of emotional pictures presented with the experimental parameters of the modified oddball paradigm.

The main aim of the present studies was to investigate the emotional ERP modulation, indexed by EPN and LPP components, as a function of the valence of preceding images. To this end, stimuli were presented in streams of six pictures, in which a sequence of pleasant, neutral, or unpleasant context pictures preceded pleasant, neutral, or unpleasant target pictures (i.e., same valence context vs. opposite valence context). Studies 1 and 2 differed with respect to the presentation of the hedonic context sequences. In Study 1, picture streams containing different valence contexts alternated randomly (cf. Cacioppo et al., 1993). In Study 2,60 sequences of each valence context were presented in succession, counterbalancing their order across participants. As a result, both studies allowed a comparison of emotional picture processing in the context of the same, a neutral, or the opposite valence picture sequence. Accordingly, the first aim was to determine whether hedonic context modulates emotion processing indexed by the EPN and LPP component. On the one hand, it has been suggested that the discrimination of emotional and neutral stimuli may represent an obligatory process, which is not modulated by hedonic context (Pastor et al., 2008). On the other hand, previous research revealed hedonic context effects on emotion processing (Cacioppo et al., 1993). Furthermore, several distinct hypotheses may be derived from previous research regarding the interaction of hedonic context and current picture processing. For instance, from an affective priming perspective, congruent valence contexts may facilitate the processing of emotional pictures if they are of the same valence as the hedonic context (cf. Avero and Calvo, 2006). Alternatively, if an emotional picture is incongruent with the hedonic context, it may be particularly salient and accordingly efficient in drawing attentional resources (Cacioppo et al., 1993). Furthermore, repeatedly presenting pictures from the same valence category may lead to sensitization effects, particularly for the processing of unpleasant images, as seen in the EMG corrugator response (Bradley et al., 1996). Accordingly, if the context would modulate processing of current emotional stimuli, the second goal of the study was to reveal the direction of this modulation with respect to the EPN and LPP components (general sensitization vs. facilitation by the same or opposite hedonic context).

\section{Methods}

\subsection{Participants}

Participants in Study 1 were seventeen ( 11 females) students from the University of Greifswald. Participants were between the ages of 19 and 26 years. In Study 2, participants were a second group of seventeen (11 females) students from the University of Greifswald, who were between the ages of 19 and 27 years. All participants received course credit towards their research requirements and provided written informed consent for the protocol, which was approved by the Review Board of the University of Greifswald.

\subsection{Stimulus materials and design}

Sixty target pictures were taken from the International Affective Picture System (IAPS; see Lang et al. 2008) depicting 20 unpleasant scenes (e.g., spiders, mutilations), 20 pleasant scenes (e.g., attractive infants, opposite sex nudes), and 20 neutral scenes (e.g., neutral faces, household objects). ${ }^{1}$ The three categories differed significantly from each other in their normative valence ratings $(M=7.3,4.9$, and 2.3 for pleasant, neutral, and unpleasant contents, respectively; scale range: 1-9). Mean arousal levels for both emotional categories were significantly higher than for neutral contents $(M=5.4,2.8$, and 6.8 for pleasant, neutral, and unpleasant contents, respectively). These target pictures were the focus of the analysis and were presented embedded in a sequence of context pictures.

A different set of 50 pleasant, 50 neutral, and 50 unpleasant IAPS pictures was selected to create the hedonic contexts. The three context categories differed significantly from each other in their normative valence ratings $(M=7.2,5.4$, and 2.5 for pleasant, neutral, and unpleasant contents, respectively). Mean arousal levels for both emotional categories were significantly higher than for neutral contents ( $M=5.3,3.4$, and 5.8 for pleasant, neutral, and unpleasant contents, respectively).

The pictures were presented in streams of 6 pictures (cf. Cacioppo et al., 1993). Five of the six pictures were drawn from the context picture set. The target picture was drawn from the target picture set and appeared third, fourth, or fifth within the picture stream. Presenting pictures from one of the three valence categories realized the pleasant, neutral, or unpleasant hedonic context. Furthermore, pictures subsequent to target pictures varied in valence to increase the likelihood that participants paid attention to the picture streams (Cacioppo et al., 1993, 1994). A total of 180 distinct picture streams occurred since each stimulus of the target picture set $(N=60)$ was presented within each of the three hedonic context categories $(N=20$ for each of the nine context by target valence combinations). The participants pressed a button to initiate each of the 180 picture streams. Each picture was displayed for $1.5 \mathrm{~s}$ and preceded by a warning $\operatorname{dot}(.5 \mathrm{~s})$ to ensure that the participants were attending to the screen. After picture offset, participants were asked to rate the valence of the pictures using a three-way response button. The inter-trial interval was $3 \mathrm{~s}$. One stream of 6 pictures served as a practice trial.

Study 1 and Study 2 differed in the arrangement of the hedonic context sequences. In Study 1, picture streams containing pleasant, neutral, or unpleasant context sequences were presented in random order. In Study 2, context picture valence was blocked so that the sixty streams of each context picture valence were presented in succession. This resulted in 3 consecutive experimental blocks, each consisting of 60 picture streams with pleasant, neutral, or unpleasant context sequences, respectively. The order of presentation was counterbalanced across participants in Study 2.

\subsection{ERP recordings and analysis}

Brain and ocular scalp potential fields were measured with a 129 lead geodesic sensor net, on-line bandpass filtered from 0.1 to $100 \mathrm{~Hz}$, and sampled at $250 \mathrm{~Hz}$ using Netstation acquisition software and EGI amplifiers (Electrical Geodesics, Inc., Eugene, OR). Electrode impedance was kept below $30 \mathrm{k} \Omega$, as recommended for this type of amplifier by EGI guidelines. Data were recorded continuously with the vertex sensor as the reference electrode. A $35 \mathrm{~Hz}$ digital low pass filter was applied off-line to the continuous EEG data. Single-trial epochs were corrected for vertical and horizontal eye movements (Miller et al., 1988). Data editing and artifact rejection were based on an elaborate method for statistical control of artifacts, specifically tailored for the analyses of dense sensor ERP recordings (Junghöfer et al., 2000). Data were baseline-corrected ( $100 \mathrm{~ms}$ prestimulus) and converted to an average reference. Finally, separate average waveforms were calculated for the 9 experimental cells (Target Valence by Context Valence) for each sensor and participant. Applying strict artifact criteria, $8.7(\mathrm{SD}=1.6)$ and $9.8(\mathrm{SD}=1.4)$ trials were used to calculate average waveforms in Study 1 and Study 2, respectively. Trial numbers were not

1 The IAPS slide numbers were as follows: pleasant - 4670, 4658, 4660, 4690, 4650, 4680, 4651, 4652, 4664, 4659, 2311, 2341, 2165, 2170, 2050, 2080, 2360, 2070, 2340, 2160: neutral - 2850, 2570, 2440, 2480, 2381, 2230, 2210, 2200, 2190, 9070, 7020, $7175,7235,7233,7010,7030,7080,7040,7002,7009$ : unpleasant - 1201, 1120 , $1300,1050,1930,3530,6510,6260,6350,6540,9405,3130,3080,3110,3060$, $3102,3053,3000,3071,3010$. 


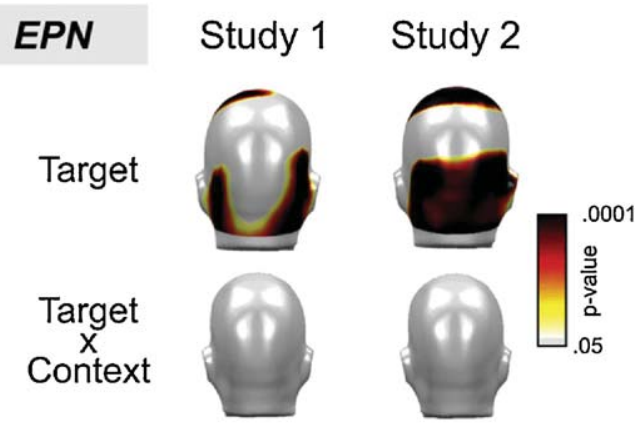

\section{LPP \\ Target \\ Target
$x$
Context}

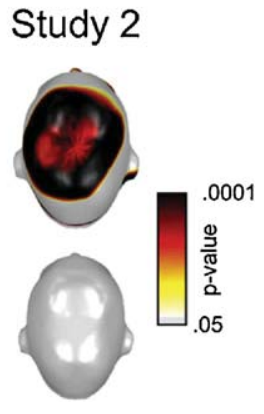

Fig. 1. Illustration of the statistical results ( $p$-value significance maps) of the pointby-point waveform ANOVA, averaged for illustrative purpose across relevant time windows for the EPN (200-300 ms; back view) and LPP (400-700 ms; top view) component for Study 1 and Study 2, respectively.

different for the target valence categories across hedonic contexts. A control analysis with liberal artifact criteria (mean trial number $=17.3$ and 17.6 for Study 1 and Study 2, respectively) revealed comparable results.

A two-step procedure was used to identify relevant ERP components. These were subsequently assessed by conventional ERP analyses based on area scores, i.e., mean activity in selected sensor regions and time windows. In a first step, visual inspection and single sensor waveform analysis were used in concert to identify relevant ERP components. To replicate previous emotion effects and explore hedonic context effects, single sensor waveform analyses were calculated for each sensor and time point separately including the factors Target Valence (pleasant vs. neutra vs. unpleasant) and Context Valence (pleasant vs. neutral vs. unpleasant). To correct for multiple testing, effects were only considered meaningful, when the effects were observed for at least eight continuous data points ( $32 \mathrm{~ms}$ ) and two neighboring sensors (Sabbagh and Taylor, 2000). Previous findings regarding the emotional modulation of the EPN and LPP component were fully replicated (see Fig. 1). However, neither single sensor waveform analysis nor visual inspection indicated effects involving hedonic context valence.

In a second step, the mean activity across selected sensor sites and time bins was calculated to score ERP components. With regard to the EPN, the mean activity over a time interval from 200 to $300 \mathrm{~ms}$ was calculated in left and right temporooccipital sensor clusters (EGI sensor numbers of the left cluster: 57, 58, 59, 60, 63, $64,65,66,67,69,70,71,72,74,75$; right cluster: $77,78,83,84,85,86,89,90$, $91,92,95,96,97,100,101)$. Due to volume conduction effects, emotion effects over occipito-temporal areas were observed with reversed polarity over anterior sites (cf. Schupp et al., 2003). Exploring these centro-frontal sensor sites mirrored the effects observed for the occipital negativity and, for brevity, will not be reported here. To assess the LPP component, the mean activity in left and right centro-parietal sensor clusters (left cluster: 7, 13, 30, 31, 32, 37, 38, 43, 53, 54; right cluster: 80, 81, 88, 87 , $94,105,106,107,112,113$ ) was calculated in a time interval from 400 to $700 \mathrm{~ms}$. EPN and LPP data were entered into a four-factorial ANOVA including the betweensubjects factor Study (Study 1: random context vs. Study 2: blocked context) and the within-subjects factors Context Valence (pleasant, neutral, unpleasant), Target Valence (pleasant, neutral, unpleasant), and Laterality (left, right).

For effects involving repeated measures, the Greenhouse-Geisser procedure was used to correct for violations of sphericity.

\section{Results}

\subsection{EPN component}

The main findings with regard to the EPN component are illustrated in Figs. 1 and 2. The single sensor waveform analysis revealed

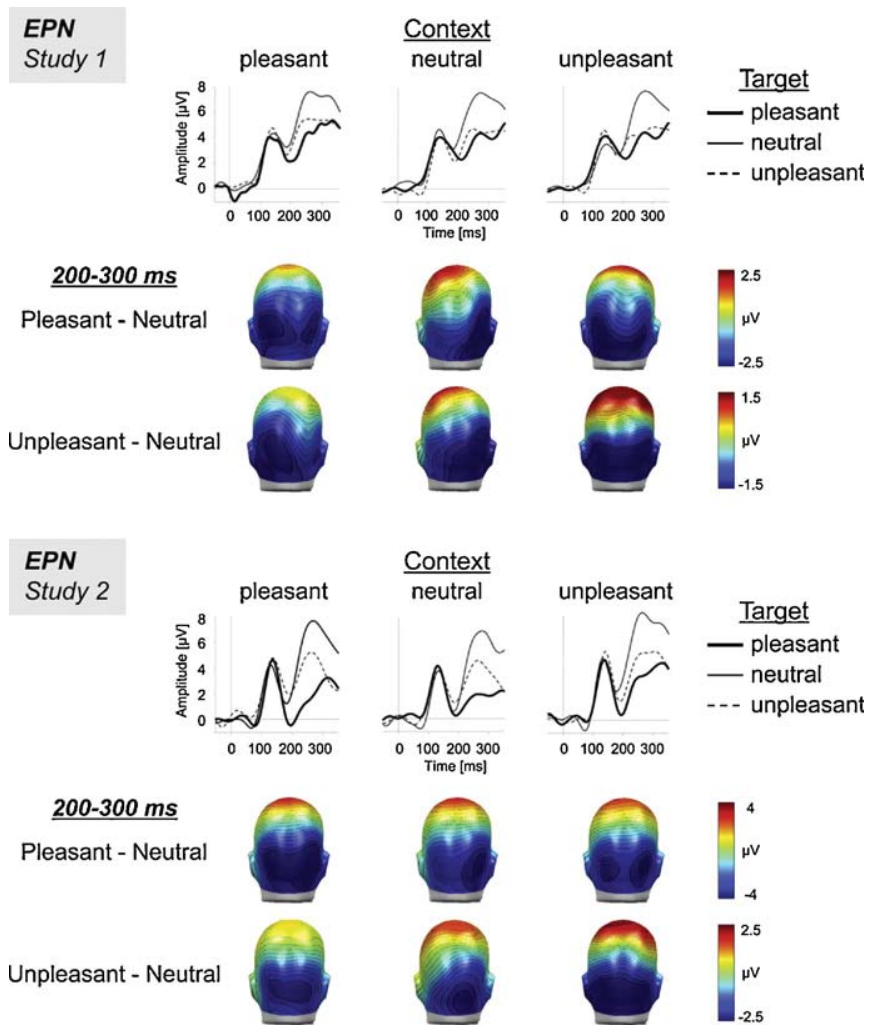

Fig. 2. Illustration of the EPN component showing a representative right occipital sensor (EGI\# 91) and scalp potential maps of the difference waves 'Pleasant Neutral', 'Unpleasant - Neutral' for the three hedonic context conditions (pleasant, neutral, and unpleasant) for Study 1 (upper panel) and Study 2 (lower panel). A back view of the model head is shown.

highly significant effects of Target Valence over occipito-temporal regions in both Study 1 and Study 2. As in previous research, the differential processing of emotional as compared to neutral pictures was seen as relative negativity over posterior regions. The effect is illustrated in Fig. 2 with regard to the temporal information by displaying a representative right occipital sensor and with regard to the topographical distribution by displaying difference maps (pleasant - neutral and unpleasant - neutral) averaged across a time interval from 200 to $300 \mathrm{~ms}$. Most importantly, the single sensor waveform analysis provided no indication of a significant interaction between Target Valence and Context Valence. Specifically, as shown also in Fig. 2, the hedonic context (pleasant, neutral, and unpleasant pictures) showed no reliable effects on emotional stimulus processing as indexed by the EPN component. Statistical analysis based on area scores over occipito-temporal regions in a time window from 200 to $300 \mathrm{~ms}$ corroborated these findings.

Replicating previous findings, a highly significant main effect of Target Valence was observed, $F(2,64)=87.5, p<.0001$. However, the effect of Target Valence was qualified by a significant interaction of Target Valence by Study $F(2,64)=3.8, p<.05$. Accordingly, separate analyses were conducted for Studies 1 and 2 . Both studies revealed a highly significant main effect of Target Valence, $F(2$, 32 ) $=34.2$ and 55.2, $p$ 's <.001, for Study 1 and Study 2, respectively. Pleasant images elicited a larger EPN component compared to neutral images, $t(16)=7.2$ and 9.4, $p$ 's $<.0001$. Similarly, unpleasant pictures elicited a larger posterior negativity as compared to neutral pictures, $t(16)=5.3$ and 5.0, $p$ 's $<.0001$. Finally, as in previous research, pleasant pictures also elicited a more negative potential compared to unpleasant pictures, $t(16)=2.9$ and 6.6, $p$ 's $<.05$.

No further effect reached significance in this analysis. Of particular interest, neither the interaction Target Valence $\times$ Context Valence, 


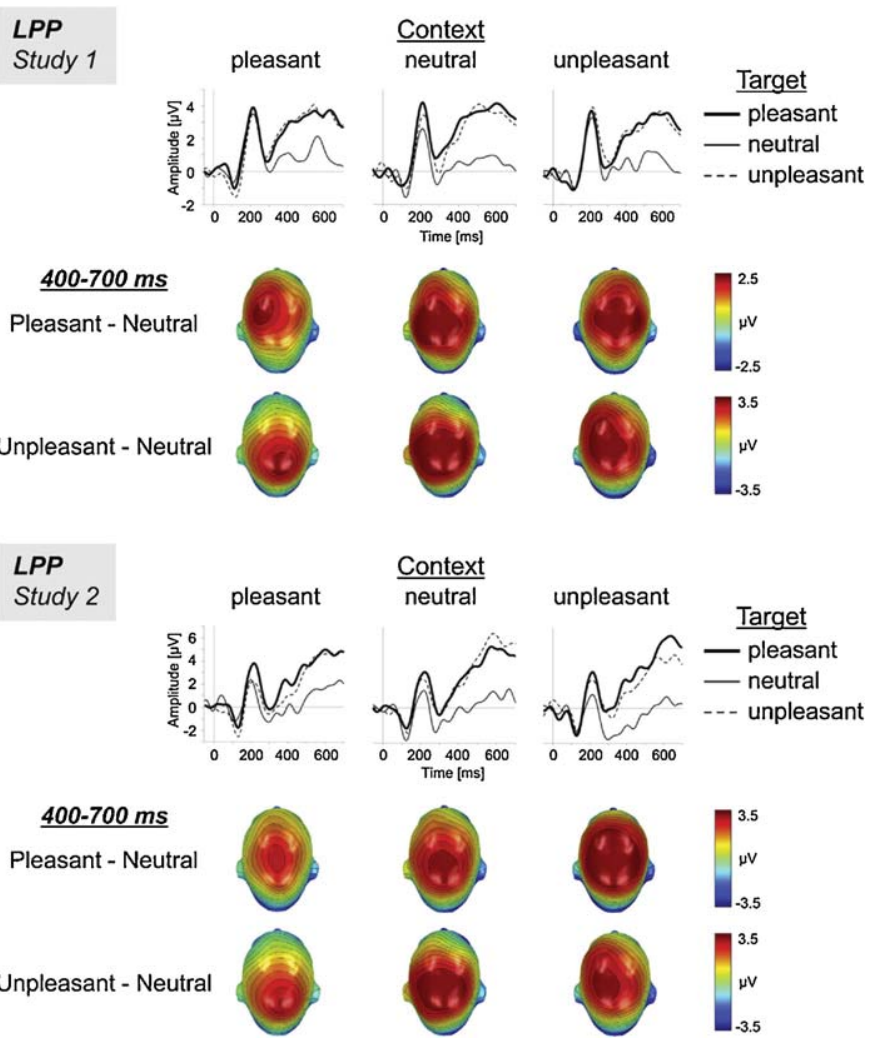

Fig. 3. Illustration of the LPP component showing a representative central sensor (EGI\# 32) and scalp potential maps of the difference waves 'Pleasant - Neutral', 'Unpleasant - Neutral' for the three hedonic context conditions (pleasant, neutral, and unpleasant) for Study 1 (upper panel) and Study 2 (lower panel). A top view of the model head is shown.

$F(4,128)=1.4$, nor the interaction Study $\times$ Target Valence $\times$ Context Valence, $F(4,128)=0.4$, approached significance. Subsequent exploratory analyses were undertaken to examine the critical interaction of Target Valence $\times$ Context Valence separately for both studies. The interaction Target Valence $\times$ Context Valence, $F(4$, 64) $=0.3$ and 0.7, was not significant in Study 1 or Study 2 .

\subsection{LPP component}

The main findings with regard to the LPP component are illustrated in Figs. 1 and 3. The outcome of the single sensor waveform revealed highly significant effects of Target Valence over centroparietal regions for both studies. As in previous research, the differential processing of emotional, compared to neutral, pictures was seen as a positive potential shift between 400 and $700 \mathrm{~ms}$. Similar to the EPN component, the single sensor waveform analysis did not reveal a significant interaction between Target Valence and Context Valence, neither in Study 1, nor in Study 2. Specifically, the emotional LPP modulation appeared similarly pronounced in pleasant, neutral, and unpleasant context blocks. To corroborate these findings, the LPP component was scored over centro-parietal regions in a time window from 400 to $700 \mathrm{~ms}$ and submitted to ANOVA analysis.

A highly significant main effect of Target Valence was observed, $F(2,64)=98.2, p<.0001$. Pleasant and unpleasant images elicited an increased positivity compared to neutral images, $t(32)=10.9$ and 11.5, $p$ 's $<.0001$, respectively. LPPs to pleasant and unpleasant images were not significantly different, $t(32)=0.7$.

No further effect reached significance in this analysis. Neither the interaction Target Valence $\times$ Context Valence, $F(4,128)=1.8$, nor the interaction Study $\times$ Target Valence $\times$ Context Valence, $F(4$,
$128)=1.3$, approached significance. Furthermore, the separate analysis of Studies 1 and 2 revealed no significant interaction of Target Valence $\times$ Context Valence, $F(4,64)=0.3$ and 1.9 , respectively.

\section{Discussion}

Emotional stimuli are discriminated from neutral contents early in the processing stream. It has been suggested that the EPN and LPP components reflect the motivational guidance of selective visual attention towards emotional cues (Schupp et al., 2006). Both studies provided a one-to-one replication of previous findings regarding the selective processing of emotional pictures. Between 200 and $300 \mathrm{~ms}$, pleasant and unpleasant pictures were associated with a relative negative potential difference over occipito-temporal sensor sites compared to neutral pictures. Furthermore, between 400 and $700 \mathrm{~ms}$, the LPP component over centro-parietal sensor sites was larger for emotional compared to neutral pictures. The novel finding of the present studies is that these effects were observed regardless of the valence of the preceding context pictures. Accordingly, the attention capture of emotional pictures seems to depend on the stimulus characteristics of the actual picture irrespective of the hedonic context of the sequence of the preceding pictures.

The current findings confirm and extend previous findings that ERPs elicited by emotional pictures are not affected by the hedonic context provided by preceding images. Pastor and colleagues (2008) presented emotional pictures for $12 \mathrm{~s}$ with a lengthy ITI interval (12s) in which picture valence varied either randomly (group 1) or was repeated across 8 presentations (group 2). The main finding was that the LPP and positive slow wave components elicited by emotional images were similar in random and repeated presentations. In comparison, the experimental paradigm used in the present studies differed in several regards. Pictures were presented in distinct, self-paced streams of six images at faster presentation rates. The repeated presentation (60 streams) of the same hedonic context in Study 2 should have facilitated the observation of hedonic context effects. Furthermore, rather than comparing hedonic context effects to a mixed series of emotional and neutral pictures, the present study presented emotional pictures within a hedonic context sequence which was either affectively congruent, incongruent, or neutral. Despite these notable methodological differences, the LPP component elicited by emotional (pleasant and unpleasant) images was similar when presented in a stream incorporating affectively congruent or incongruent context pictures. In addition, the same pattern of results was observed with regard to the EPN component, which reflects a relatively early transient processing stage at which emotional stimuli are tagged based on their significance (Schupp et al., 2006). While the pattern of larger EPNs to pleasant and unpleasant compared to neutral pictures (independent from hedonic context) was highly significant in both studies, the effect appeared even more pronounced in Study 2. This unexpected finding awaits replication in future studies. Overall, there is accumulating evidence that the discrimination of emotional and neutral stimuli represents an obligatory process during initial stimulus categorization.

Hedonic context effects on emotion processing have been examined in a separate line of research using rapid serial presentation paradigms. In one study, emotional and neutral pictures were presented in a rapid stream with no inter-stimulus interval $(330 \mathrm{~ms}$; Flaisch et al., 2008a). Two main effects were observed. As expected, emotional pictures elicited a larger EPN component compared to neutral images. Of particular interest, temporal interference effects were observed in that the processing of the current picture (irrespective of its valence) was attenuated when preceded by an emotional (pleasant or unpleasant) picture. A further study, which presented the images for $660 \mathrm{~ms}$, revealed similar effects for the 
LPP component (Flaisch et al., 2008b). Specifically, the LPP elicited by the current picture was attenuated when preceded by an emotional rather than by a neutral image. No interference effects were observed for the EPN or LPP component in the present study, which presented the pictures longer $(1.5 \mathrm{~s})$ and at slower rate $(\mathrm{ITI}=3 \mathrm{~s})$. Thus, the present findings provide boundary information regarding temporal interference effects. Competition for processing resources between the current and preceding image may thus be limited to conditions with high demands on perceptual processing.

The problems associated with the interpretation of null-findings need to be acknowledged. It is possible that larger samples may reveal hedonic context effects. However, as the current study did not observe any hedonic context effects, it seems safe to conclude that potential hedonic context effects are minor in comparison to the pronounced effect observed for target picture valence in the present studies. Furthermore, hedonic context effects may be observed by relying on other ways to create hedonic context or by using different stimulus types (cf. Cacioppo et al., 1993, 1994; Crites et al., 1995). In the present studies, hedonic context was created by presenting picture stimuli from a variety of distinct categories of human experience (Bradley et al., 2001). However, hedonic context effects may be seen when images from a single semantic category are presented (i.e., erotica, babies, mutilations, or animal threat). The study of hedonic context effects may furthermore be informative with regard to clinical disorders such as the spectrum of anxiety disorders or drug addiction. Previous studies have already revealed the enhanced processing of drug- and fear-relevant stimuli in drug addiction and small animal phobia, respectively (e.g., Franken et al., 2008; Michalowski et al., 2009; Miltner et al., 2005; Kopp and Altmann, 2005). The repeated presentation of disorder-relevant stimuli may provide complementary information regarding possible sensitization effects to disorder-relevant stimuli revealing additional neural systems not engaged in healthy subjects. Overall, while there is yet no evidence for hedonic context effects on IAPS picture processing, future studies may reveal such effects by investigating different ways to create hedonic context and selected clinical samples.

In contrast to the ERP findings, previous research examining hedonic context effects on emotion processing revealed significant effects for motor response measures. For instance, Bradley and colleagues (1996) presented continuous series of pleasant, neutral, or unpleasant pictures and observed sensitization effects for the corrugator response during unpleasant picture viewing. Furthermore, Pastor and colleagues (2008) observed that cardiac deceleration, a motor indicator of attentional orienting, was attenuated in blocked compared to mixed picture presentations. These studies used rather long picture presentation times raising the issue whether hedonic context effects for motor responses rely on more sustained picture processing. However, a further study observed unpleasant context effects on the startle reflex even when the pictures were presented as rapid and continuous stream (Smith et al., 2006). Accordingly, hedonic context effects may differ between measures of perceptual processing and stimulus evaluation on the one hand and somatic and autonomic motor responses on the other (cf. Cacioppo et al., 1999). A broader perspective on emotion processing, including the consideration of the social context and the measurement of cortical and peripheral-physiological measures seems informative for the understanding of psychopathology, in particular the anxiety spectrum. Tracking the flow of information processing from initial stimulus evaluation to the motor output stage would allow to determine the specific stage of processing at which stimulus processing of feared objects is sensitized by anticipatory anxiety.

Overall, there is increasing evidence that the emotional guidance of attention is dictated by the information depicted in the current stimulus. Studies investigating the effects of repeated stimulus exposure and hedonic context support this hypothesis (Codispoti et al., 2007; Ferrari et al., 2011; Flaisch et al., 2008a,b; Pastor et al., 2008; Schupp et al., 2006). While implicit and instructed stimulus significance are often associated with similar ERP modulations in terms of an occipital negativity and a late positive potential, a recent study observed distinct effects for both mechanisms of attention regulation (Bublatzky and Schupp, 2011). Using the instructed fear paradigm, participants were verbally instructed that they might receive an electric shock when a specific emotional (pleasant or unpleasant) or neutral image cue was presented. Instructed fear signals elicited a rather distinct ERP signature (P1, P2, and LPP) which was independent from the EPN and LPP modulations associated with emotional stimulus contents. Thus, while stimulus detection tasks often show similar ERP modulations to emotion processing (Ferrari et al., 2008; Schupp et al., 2007), intrinsic and instructed stimulus significance can also be dissociated from each other at the level of stimulus encoding. The obligatory nature of the attention capture by emotional cues may also have implications for psychotherapy research. Specifically, increased selective attention to pathology relevant cues is often observed (e.g., increased selective attention to phobia-relevant stimuli) and interpreted as an attention problem (Mathews and MacLeod, 1994). However, the regulation of the facilitated processing of emotional and task-relevant stimuli is presumably at least in part subserved by distinct neural circuits. Emotional stimuli engage cortical and subcortical limbic structures (Lang and Davis, 2006), which, in addition to modulating cortical attention systems (Posner and Peterson, 1990), may regulate perceptual processing in visual cortex by direct projections (Emery and Amaral, 2000) or via ascending neuromodulatory systems (Derryberry and Tucker, 1991). Considering such neural differences in terms of attentional control mechanisms may help to understand why cognitive interventions attempting to change selective attention to pathology relevant cues might not be as effective than stimulus driven approaches (e.g., exposure based interventions) aiming to change the emotional impact of these cues by extinction learning.

\section{Acknowledgements}

This research was supported by the German Research Foundation (Schu 1074/10-3 and 1074/11-2). We would like to thank Dr. Jessica Stockburger for help in data acquisition and analysis.

\section{References}

Avero, P., Calvo, M.G., 2006. Affective priming with pictures of emotional scenes: the role of perceptual similarity and category relatedness. Spanish Journal of Psychology 9, 10-18.

Bradley, M.M., Cuthbert, B.N., Lang, P.J., 1996. Picture media and emotion: effects of a sustained affective context. Psychophysiology 33, 662-670.

Bradley, M.M., Codispoti, M., Cuthbert, B.N., Lang, P.J., 2001. Emotion and motivation $\mathrm{I}$ : defensive and appetitive reactions in picture processing. Emotion 1, 276-298.

Bradley, M.M., Sabatinelli, D., Lang, P.J., Fitzsimmons, J.R., King, W.M., Desai, P., 2003. Activation of the visual cortex in motivated attention. Behavioral Neuroscience $117,369-380$

Bradley, M.M., 2009. Natural selective attention: orienting and emotion. Psychophysiology 46, 1-11.

Bublatzky, F., Schupp, H.T., 2011. Pictures cueing threat: brain dynamics in viewing explicitly instructed danger cues. Social Cognitive \& Affective Neuroscience, http://dx.doi.org/10.1093/scan/nsr032.

Cacioppo, J.T., Crites, S.L., Berntson, G.G., Coles, M.G., 1993. If attitudes affect how stimuli are processed should they not affect the event-related brain potential? Psychological Science 4,108-112.

Cacioppo, J.T., Crites, S.L., Gardner, W.L., Berntson, G.G., 1994. Bioelectrical echoes from evaluative categorizations: I. A late positive brain potential that varies as a function of trait negativity and extremity. Journal of Personality and Social Psychology 67, 115-125.

Cacioppo, J.T., Gardner, W.L., Berntson, G.G., 1999. The affect system has parallel and integrative processing components: form follows function. Journal of Personality and Social Psychology 76, 839-855. 
Codispoti, M., Ferrari, V., Bradley, M.M., 2007. Repetition and ERPs: distinguishing early and late processes in affective picture perception. Journal of Cognitive Neuroscience 19, 577-586.

Costafreda, S.G., Brammer, M.J., David, A.S., Fu, C.H., 2008. Predictors of amygdala activation during the processing of emotional stimuli: a meta-analysis of 385 PET and fMRI studies. Brain Research Reviews 58, 57-70.

Crites, S.L., Cacioppo, J.T., Gardner, W.L., Berntson, G.G., 1995. Bioelectrical echoes from evaluative categorization: II. A late positive brain potential that varies as a function of attitude registration rather than attitude report. Journal of Personality and Social Psychology 68, 997-1013.

Derryberry, D., Tucker, D.M., 1991. The adaptive base of the neural hierarchy: elementary motivational controls on network function. In: Dienstbier, R.A. (Ed.), Nebraska Symposium on Motivation, 1990: Perspectives on Motivation. University of Nebraska Press, Lincoln, NE, USA, pp. 289-342.

Emery, N.J., Amaral, D.G., 2000. The role of the amygdala in primate social cognition. In: Lane, R.D., Nadel, L. (Eds.), Cognitive Neuroscience of Emotion. Oxford University Press, New York, NY, USA, pp. 156-191.

Ferrari, V., Codispoti, M., Cardinale, R., Bradley, M.M., 2008. Directed and motivated attention during processing of natural scenes. Journal of Cognitive Neuroscience $20,1753-1761$

Ferrari, V., Bradley, M.M., Codispoti, M., Lang, P.J., 2011. Repetitive exposure: brain and reflex measures of emotion and attention. Psychophysiology 48, 515-522.

Flaisch, T., Junghöfer, M., Bradley, M.M., Schupp, H.T., Lang, P.J., 2008a. Rapid picture processing: affective primes and targets. Psychophysiology 45, 1-10.

Flaisch, T., Stockburger, J., Schupp, H.T., 2008b. Affective prime and target picture processing: an ERP analysis of early and late interference effects. Brain Topography 20, 183-191.

Flaisch, T., Schupp, H.T., Renner, B., Junghöfer, M., 2009. Neural systems of visual attention responding to emotional gestures. Neuroimage 45, 1339-1346.

Flaisch, T., Häcker, F., Renner, B., Schupp, H.T., 2011. Emotion and the processing of symbolic gestures: an event-related brain potential study. Social Cognitive and Affective Neuroscience 6, 109-118.

Franken, I.H.A., Dietvorst, R.C., Hesselmans, M., Franzek, E.J., Van De Wetering, B.J.M. Van Strien, J.W., 2008. Cocaine craving is associated with electrophysiological brain responses to cocaine-related stimuli. Addiction Biology 13, 386-392.

Junghöfer, M., Bradley, M.M., Elbert, T.R., Lang, P.J., 2001. Fleeting images: a new look at early emotion discrimination. Psychophysiology 38, 175-178.

Junghöfer, M., Elbert, T.R., Tucker, D.M., Rockstroh, B., 2000. Statistical control of artifacts in dense array EEG/MEG studies. Psychophysiology 37, 523-532.

Junghöfer, M., Sabatinelli, D., Bradley, M.M., Schupp, H.T., Elbert, T.R., Lang, P.J., 2006. Fleeting images: rapid affect discrimination in the visual cortex. Neuroreport 17 , 225-229.

Kissler, J., Assadollahi, R., Herbert, C., 2006. Emotional and semantic networks in visual word processing: insights from ERP studies. Progress in Brain Research 156, 147-183.

Kopp, B., Altmann, R., 2005. Neurocognitive effects of phobia-related stimuli in animal-fearful individuals. Cognitive Affective \& Behavioral Neuroscience 5, 373-387.

Lang, P.J., Bradley, M.M., Cuthbert, B.N., 1997. Motivated attention: affect, activation, and action. In: Lang, P.J., Simons, R.F., Balaban, M. (Eds.), Attention and Emotion: Sensory and Motivational Processes. Erlbaum, Mahwah, NJ, pp. 97-135.

Lang, P.J. Davis, M.E., 2006. Emotion, motivation and the brain: reflex foundations in animal and human research. Progress in Brain Research 156, 3-29.

Lang, P.J., Bradley, M.M., Cuthbert, B.N., 2008. International affective picture system (IAPS): affective ratings of pictures and instruction manual. Technical Report A-8. University of Florida, Gainesville, FL.

Mathews, A.M., MacLeod, C., 1994. Cognitive approaches to emotion and emotional disorders. Annual Review of Psychology 45, 25-50.
Miller, G.A., Gratton, G., Yee, C.M., 1988. Generalized implementation of an eye movement correction procedure. Psychophysiology 25, 241-243.

Michalowski, J.M., Melzig, C.A., Weike, A.I., Stockburger, J., Schupp, H.T., Hamm, A.O., 2009. Brain dynamics in spider phobic individuals exposed to phobia-relevant or other emotional stimuli. Emotion 9, 306-315.

Miltner, W.H., Trippe, R.H., Krieschel, S., Gutberlet, I., Hecht, H., Weiss, T., 2005. Event-related brain potentials and affective responses to threat in spider/snakephobic and non-phobic subjects. International Journal of Psychophysiology 57, 43-52.

Mühlberger, A., Wieser, M.J., Herrmann, M.J., Weyers, P., Tröger, C., Pauli, P., 2009. Early cortical processing of natural and artificial emotional faces differs between lower and higher socially anxious persons. Journal of Neural Transmission 116, 735-746.

Öhman, A., Flykt, A., Lundqvist, D., 2000. Unconscious emotion: evolutionary perspectives, psychophysiological data and neuropsychological mechanisms. In: Lane, R.D., Nadel, L. (Eds.), Cognitive Neuroscience of Emotion. Oxford University Press, New York, pp. 296-327.

Palomba, D. Angrilli, A., Mini, A., 1997. Visual evoked potentials, heart rate responses and memory to emotional pictorial stimuli. International Journal of Psychophysiology 27, 55-67.

Pastor, M.C., Bradley, M.M., Low, A., Versace, F., Molto, J., Lang, P.J., 2008. Affective picture perception: emotion, context, and the late positive potential. Brain Research 1189, 145-151.

Posner, M.I., Peterson, S.E., 1990. The attention system of the human brain. Annual Review of Neuroscience 13, 25-42.

Sabatinelli, D., Lang, P.J., Keil, A., Bradley, M.M., 2007. Emotional perception: correlation of functional MRI and event related potentials. Cerebral Cortex 17, 1085-1091.

Sabatinelli, D., Fortune, E.E., Li, Q., Siddiqui, A., Krafft, C., Oliver, W.T., Beck, S., Jeffries, J., 2011. Emotional perception: meta-analyses of face and natural scene processing. Neuroimage 54, 2524-2533.

Sabbagh, M.A., Taylor, M., 2000. Neural correlates of theory-of-mind reasoning: an event-related potential study. Psychological Science 11, 46-50.

Sato, W., Kochiyama, T., Yoshikawa, S., Matsumura, M., 2001. Emotional expression boosts early visual processing of the face: ERP recording and its decomposition by independent component analysis. Neuroreport 12, 709-714.

Schupp, H.T., Cuthbert, B.N., Bradley, M.M., Cacioppo, J.T., Ito, T., Lang, P.J., 2000. Affective picture processing: the late positive potential is modulated by motivational relevance. Psychophysiology 37, 257-261.

Schupp, H.T., Junghöfer, M., Weike, A.I., Hamm, A.O., 2003. Emotional facilitation of sensory processing in the visual cortex. Psychological Science 14, 7-13.

Schupp, H.T., Öhman, A., Junghöfer, M., Weike, A.I., Stockburger, J., Hamm, A.O., 2004. The facilitated processing of threatening faces: an ERP analysis. Emotion 4, 189-200.

Schupp, H.T., Flaisch, T., Stockburger, J., Junghöfer, M., 2006. Emotion and attention: event-related brain potential studies. Progress in Brain Research 156, $31-51$

Schupp, H.T., Stockburger, J., Codispoti, M., Junghofer, M., Weike, A.I., Hamm, A.O., 2007. Selective visual attention to emotion. Journal of Neuroscience 27, 1082-1089.

Smith, J.C., Löw, A., Bradley, M.M., Lang, P.J., 2006. Rapid picture presentation and affective engagement. Emotion 6, 208-214.

Van Berkum, J.J., Holleman, B., Nieuwland, M., Otten, M., Murre, J., 2009. Right or wrong? The brain's fast response to morally objectionable statements. Psychological Science 20, 1092-1099.

Wieser, M., Pauli, P., Reicherts, P., Mühlberger, A., 2010. Don't look at me in anger! Enhanced processing of angry faces in anticipation of public speaking. Psychophysiology 47, 271-280. 